Research Paper

\title{
Comparison of the Analgesic Properties of Sevoflurane and Desflurane Using Surgical Pleth Index at Equi-Minimum Alveolar Concentration
}

\author{
Kyoungho Ryu ${ }^{1}$, Keulame Song ${ }^{1}$, Jia Kim ${ }^{1}$, Eugene Kim², Seong-Hyop Kim³, 4, 5凶 \\ 1. Department of Anesthesiology and Pain Medicine, Kangbuk Samsung Hospital, Sungkyunkwan University School of Medicine, Seoul, Republic of Korea; \\ 2. Department of Orthopedic Surgery, Kangbuk Samsung Hospital, Sungkyunkwan University School of Medicine, Seoul, Republic of Korea; \\ 3. Department of Anesthesiology and Pain medicine, Konkuk University Medical Center, Konkuk University School of Medicine, Seoul, Republic of Korea; \\ 4. Department of Infection and Immunology, Konkuk University School of Medicine, Seoul, Korea; \\ 5. Department of Medicine, Institute of Biomedical Science and Technology, Konkuk University School of Medicine, Seoul, Korea. \\ $\square$ Corresponding author: Seong-Hyop Kim. Department of Anesthesiology and Pain medicine, Konkuk University Medical Center, 120-1, Neungdong-ro \\ (Hwayang-dong), Gwangjin-gu, Seoul, 05030, Republic of Korea. Tel: +82-2-2030-5454; Fax: +82-2-2030-5449. Email: yshkim75@daum.net \\ (c) Ivyspring International Publisher. This is an open access article distributed under the terms of the Creative Commons Attribution (CC BY-NC) license \\ (https://creativecommons.org/licenses/by-nc/4.0/). See http://ivyspring.com/terms for full terms and conditions.
}

Received: 2017.03.28; Accepted: 2017.06.18; Published: 2017.08.18

\begin{abstract}
Background: Traditionally, minimum alveolar concentration (MAC) has been used as the standard measure to compare the potencies of volatile anesthetics. However, it reflects the spinal mechanism of immobility rather than the subcortical mechanism of analgesia. Recently, the surgical pleth index (SPI) derived from photoplethysmographic waveform was shown to reflect the intraoperative analgesic component. This study was designed to compare the SPI values produced by equi-MAC of two commonly used volatile anesthetics, sevoflurane and desflurane.

Methods: Seventy-two patients undergoing arthroscopic shoulder surgery were randomly assigned to two groups receiving either sevoflurane $(n=36)$ or desflurane $(n=36)$. General anesthesia was maintained with the respective volatile anesthetic only. A vaporizer was adjusted to maintain end-tidal anesthetic concentration at age-corrected 1.0 MAC throughout the study period. The SPI value as an analgesic estimate and the bispectral index (BIS) value as a hypnotic estimate were recorded at predefined time points during the standardized surgical procedure.

Results: During the steady state of age-corrected 1.0 MAC, mean SPI values throughout the entire study period were significantly higher in the sevoflurane group than in the desflurane group ( $38.1 \pm 12.8$ vs. $30.7 \pm 8.8$, respectively, $P=0.005$ ), and mean BIS values were significantly higher in the sevoflurane group than in the desflurane group $(40.7 \pm 5.8$ vs. $36.8 \pm 6.2$, respectively, $P=0.008)$.

Conclusions: Equi-MAC of sevoflurane and desflurane did not produce similar surgical pleth index values. Therefore, sevoflurane and desflurane may have different analgesic properties at equipotent concentrations.
\end{abstract}

Key words: minimum alveolar concentration, volatile anesthetic, surgical pleth index.

\section{Introduction}

The minimum alveolar concentration (MAC) concept has traditionally been used to compare the potencies of volatile anesthetics [1]. However, it does not discriminate between the different components of general anesthesia (i.e., hypnosis, analgesia, and immobility) [2]. Moreover, it is generally accepted that equi-MAC of various volatile anesthetics have dissimilar hypnotic properties [3-8]. With the development of advanced techniques to measure hypnosis and immobilization, related monitoring has been routinely applied [9-11]. Nonetheless, the use of devices for monitoring analgesia has been limited. Recently, the surgical pleth index (SPI), derived from the photoplethysmographic waveform, was shown to be an objective surrogate for monitoring intraoperative analgesia [12-16]. 
Sevoflurane and desflurane are most commonly used volatile anesthetics because of their rapid pharmacokinetic properties. Ideal balanced anesthesia results from a proper combination of volatile anesthetics and anesthetic supplements such as opioids. To ensure safe administration of anesthetic supplements and early recovery from general anesthesia, it is mandatory to comprehend the differences in analgesic and hypnotic properties of various volatile anesthetics. The authors hypothesized that equi-MAC of two volatile anesthetics would not show equivalent analgesic properties. To this end, this study compared the SPI values produced by equi-MAC of two volatile anesthetics, sevoflurane and desflurane, in patients undergoing arthroscopic shoulder surgery under interscalene brachial plexus block (ISBPB).

\section{Methods}

This prospective, randomized trial was registered at clinicaltrials.gov (Identifier: NCT02609802) prior to inclusion of the first patient. After approval from the Institutional Ethics Committee (Kangbuk Samsung Hospital Institutional Review Board, Seoul, Republic of Korea; Approval number: KBSMC 2015-09-028), written informed consent was obtained from American Society of Anesthesiologists physical status classification I and II patients aged 19-65 years, undergoing arthroscopic shoulder surgery. Patients with a history of any neurological or psychiatric disease, cardiac arrhythmia, diabetes mellitus, alcohol or drug abuse, and use of any medication affecting the central nervous system or autonomic nervous system were excluded from the study.

The subjects were assigned to sevoflurane and desflurane groups to determine the maintenance volatile anesthetic at a 1:1 ratio using a random-permuted block randomization algorithm via a web-based response system (www.randomization. com). Allocation concealment was performed using serially numbered opaque envelopes, each containing a folded slip of paper on which was written the anesthesia protocol (sevoflurane or desflurane). The envelopes were stored and opened by an independent coordinator in an office distant from the hospital. The subject allocation was not changed after the envelope was opened.

No anticholinergic drugs or sedatives were administered as premedication. After arrival in the operating room, standard monitoring $\left(\mathrm{S} / 5^{\mathrm{TM}}\right.$ Anesthesia Monitor; GE Healthcare, Helsinki, Finland), including non-invasive blood pressure, electrocardiography and pulse oximetry, were applied. After the skin of the forehead had been prepared with alcohol-soaked cotton, a BIS-Quatro ${ }^{\mathrm{TM}}$ sensor (Covidien, Mansfield, MA, USA) was placed on the forehead of the side contralateral to the surgery. To standardize intra-operative surgical stimuli and reduce post-operative pain, all of the patients received pre-operative ISBPB. For ISBPB, the ultrasound-guided lateral-to-medial in-plane technique was performed using a $60 \mathrm{~mm}$ 22-gauge short-beveled needle (Unisis Corporation, Saitama, Japan). After the needle tip was placed at the correct position, $0.5 \%$ ropivacaine $(0.2 \mathrm{ml} / \mathrm{kg}$ of body weight $)$ with 1:200,000 epinephrine was injected. All of the block procedures were performed by one anesthesiologist (K. Ryu) with experience performing more than 300 ISBPBs. Before induction of general anesthesia, the sensory block was evaluated by testing cold sensation with alcohol-soaked cotton in the appropriate dermatome of the brachial plexus. The block was considered efficiently analgesic for arthroscopic shoulder surgery if it covered dermatomes $C_{3}-T_{1}$. In cases in which the block was incomplete on the test or the postoperative wound pain score using an 11-point numerical rating scale in the post-anesthesia care unit was $\geq 1$, the data for the subject were excluded from the final analysis.

General anesthesia was induced with 1\% propofol (Fresofol ${ }^{\circledR}$ MCT 1\%; Fresenius Kabi Austria $\mathrm{GmbH}$, Linz, Austria). To standardize and minimize induction dose, propofol was infused via a target-controlled infusion (TCI) device (Orchestra Base Primea ${ }^{\circledR}$; Fresenius Vial, Brezins, France) using the Marsh pharmacokinetic model. A TCI device was only used for induction of anesthesia. The initial target predicted effect-site concentration of propofol (Ce propofol) in the TCI device was set to $3.0 \mu \mathrm{g} / \mathrm{ml}$, and propofol infusion was started in flash mode. To minimize infusion dose, $\mathrm{Ce}_{\text {propofol }}$ in the $\mathrm{TCI}$ device was adjusted to $0.0 \mu \mathrm{g} / \mathrm{ml}$ immediately after loss of consciousness and propofol infusion was stopped. At the same time, randomly assigned volatile anesthetic, either sevoflurane (Sevorane ${ }^{\circledR}$; AbbVie Ltd., Maidenhead, UK) or desflurane (Suprane ${ }^{\circledR}$; Baxter Healthcare, Guayama, Puerto Rico), was given via a tight-fitting facemask, after which $0.8 \mathrm{mg} / \mathrm{kg}$ rocuronium was given for neuromuscular block and the trachea was intubated. Volatile anesthetic concentration increase was facilitated by the overpressurization technique using about 2.0 MAC with the aim of reaching 1.0 MAC. End-tidal anesthetic gas concentrations were measured continuously using the infrared spectrophotometric analyzer of an anesthesia workstation.

The vaporizer was adjusted by an independent coordinator who was blinded to the study design to maintain the end-tidal anesthetic concentration at 1.0 
MAC throughout the entire study period. The MAC value was corrected based on age-related iso-MAC charts $[17,18]$. Anesthesia was maintained with the volatile anesthetic of age-corrected 1.0 MAC as the single anesthetic. Neither opioids nor nitrous oxide was used during the entire study period. The hypnotic component of volatile anesthesia was monitored using the BIS value. If bispectral index (BIS) value $>70$, mean arterial pressure $(\mathrm{MAP})<60$ $\mathrm{mmHg}$, heart rate $(\mathrm{HR})<45$ beats/min, or HR $>120$ beats/min at any time during the study period, additional sedatives, vasopressors, vagolytics, or beta-blockers were administered, respectively, and the data for the subject were excluded from the final analysis. The operating room was kept as quiet as possible and all of the external stimuli were minimized during the study period. The level of neuromuscular block was monitored continuously by train-of-four (TOF) stimulation. In both groups, a TOF count of 1-2 was maintained during the study period. End-tidal partial pressure of carbon dioxide and esophageal temperature were monitored continuously to ensure normocarbia and normothermia, respectively.

The analgesic component of volatile anesthesia was monitored using the SPI value. Photoplethysmographic waveforms were collected from the index finger of the arm contralateral to the surgery. The SPI is a dimensionless numerical index for monitoring the nociceptive-antinociceptive
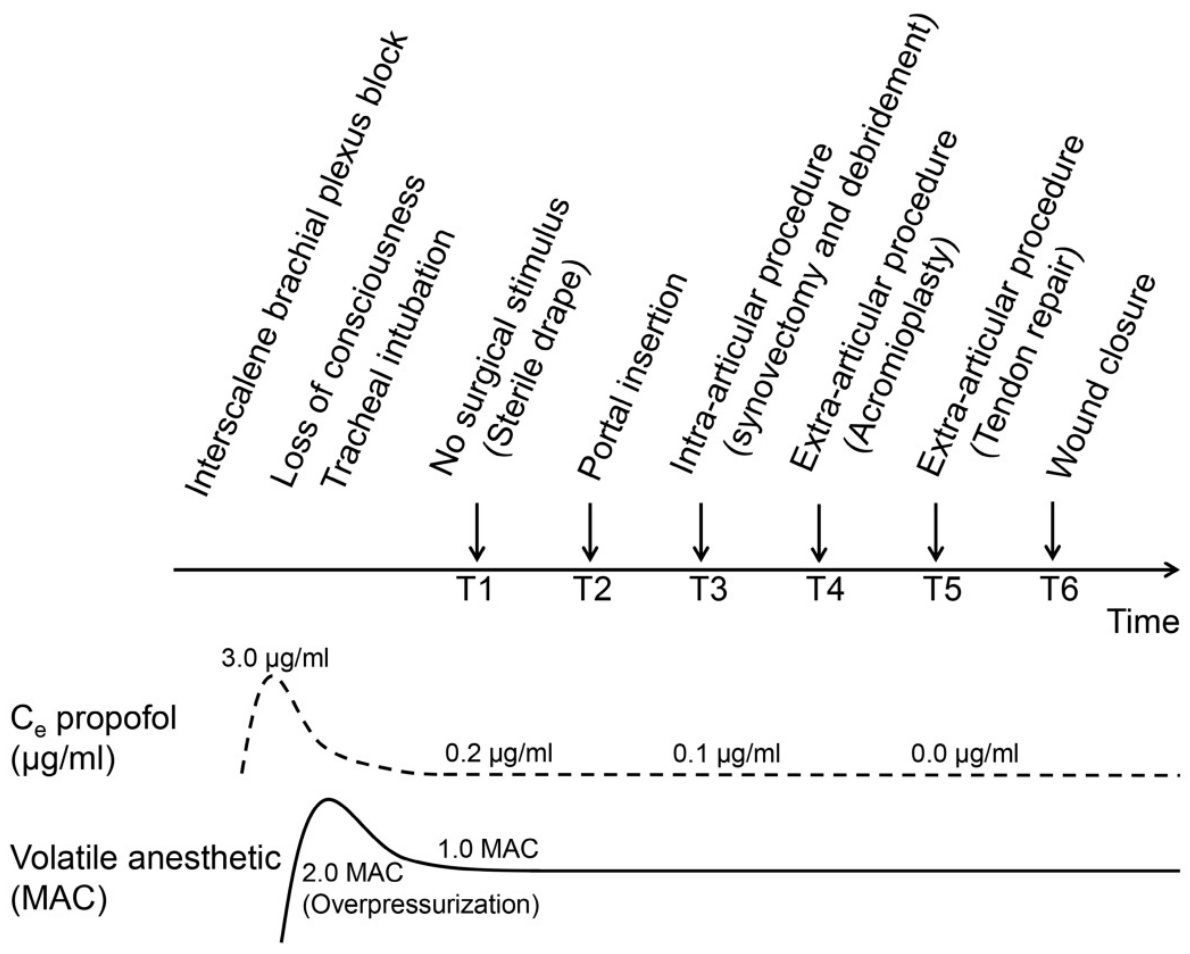

Figure 1. Study timeline. The arrows indicate time points of standardized surgical procedure at which study outcomes were recorded. The dashed line represents the predicted effect-site concentration $(\mathrm{Ce})$ of propofol; the solid line represents end-tidal anesthetic concentration. balance obtained by finger clip sensor used for measuring transcutaneous oxygen saturation. The SPI is measured by a combination of central sympathetic tone, denoted as heart beat interval (HBI), and peripheral sympathetic tone, denoted as photoplethysmographic pulse wave amplitude (PPGA). The SPI is based on an algorithm combining the normalized $\mathrm{HBI}\left(\mathrm{HBI}_{\text {norm}}\right)$ and the normalized PPGA (PPGA norm$_{\text {) }}$ data using the following equation: $\mathrm{SPI}=100-\left(0.33 \times \mathrm{HBI}_{\text {norm }}\right)+\left(0.67 \times \mathrm{PPGA}_{\text {norm }}\right)$ [12] The SPI is shown as a value from 0 (no surgical stress) to 100 (maximal surgical stress), with a value of 50 representing mean stress level during general anesthesia.

The SPI with BIS values were measured, including hemodynamic parameters, MAP and HR. All of the study outcome recordings were obtained at predefined time points during the standardized surgical procedure (Figure 1): T1 (during sterile draping, no surgical stimulus), T2 (during portal insertion), T3 (during synovectomy and debridement, intra-articular procedure), T4 (during acromioplasty, extra-articular procedure), T5 (during tendon repair, extra-articular procedure), and T6 (during wound closure at the end of surgery). The study outcomes were recorded by an investigator who was blinded to group allocation. An initial 30-minute waiting period was allowed for the effects of the induction dose of propofol to dissipate and for the transition to pure volatile anesthesia. All of the study outcomes were obtained after the $\mathrm{Ce}$ propofol of the TCI device was reduced below $0.2 \mu \mathrm{g} / \mathrm{ml}$. To ensure brain-alveolar equilibration of the anesthetic, all of the data were only recorded after meeting the steady-state period (defined as a condition in which constant end-tidal anesthetic concentration is maintained without vaporizer adjustment during at least $5 \mathrm{~min}$ ) of 1.0 MAC.

The primary outcome of the study was SPI value at a steady state of age-corrected 1.0 MAC. The sample size was calculated based on the results of a pilot study in 16 patients (eight patients per group). A sample size of 36 subjects per group was estimated to detect a mean difference in 5 points in SPI values, assuming a standard deviation of 7.5 points (based on 
a pilot study), using the two-tailed t-test of the difference between means, a power of $80 \%$ and a significance level of $5 \%$. To allow for potential dropouts and missing data, 90 patients were recruited. All of the statistical analyses were performed using PASW Statistics 18.0 (IBM, Armonk, NY, USA). All of the analyses were performed according to the initially allocated group on the basis of the intention-to-treat principle. No interim analyses were planned or performed. Data are presented as the frequency for categorical variables and the mean \pm standard deviation (SD) or median (interquartile range $[\mathrm{IQR}]$ ) for continuous variables. Baseline and clinical characteristics were compared between groups using the chi-squared test or Fisher's exact test for categorical variables and Student's $t$-test or the Mann-Whitney $U$ test for continuous variables as appropriate. Mean SPI and BIS values throughout the entire study period were compared by the between-subjects effects test using repeated-measures analysis of variance (RM-ANOVA) between groups. Values between time points in each group were compared by the within-subjects effects test of
RM-ANOVA. Values at each time point between groups were compared by Student's $t$-test. In all of the analyses, $P<0.05$ was taken to indicate statistical significance.

\section{Results}

A total of 90 patients were recruited between November 2015 and July 2016, but one patient declined to participate and 12 were ineligible based on the exclusion criteria. Therefore, 39 and 38 subjects were randomized into the sevoflurane group and the desflurane group, respectively. Three subjects were excluded from the sevoflurane group (two required intraoperative ephedrine administration, and one case had an SPI monitoring error), two subjects were excluded from the desflurane group (one required ephedrine administration and one required esmolol administration). Thus, the final analyses were confined to 72 subjects, with 36 subjects in the sevoflurane group and 36 subjects in the desflurane group (Figure 2).

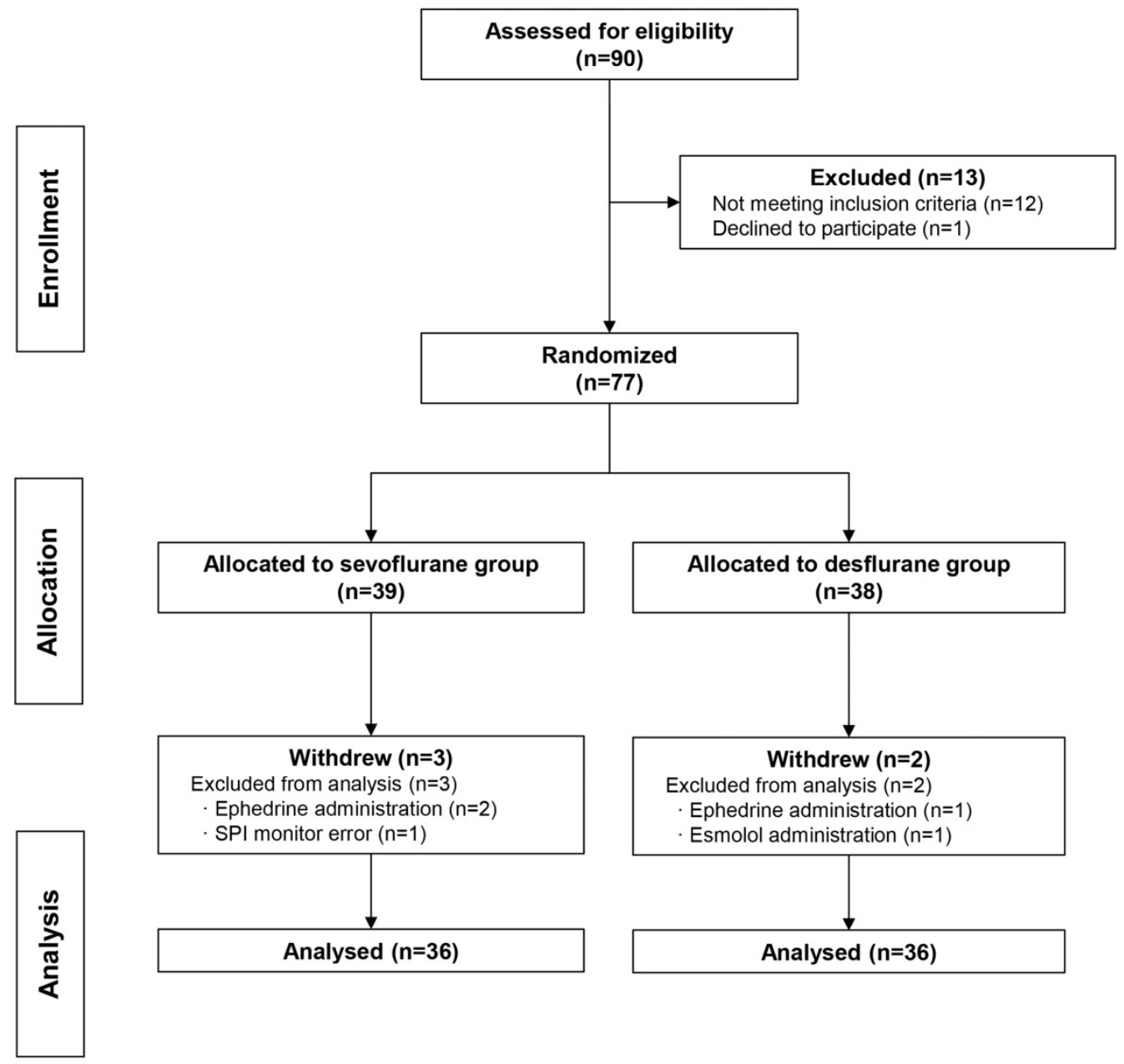

Figure 2. CONSORT flow diagram. Enrolment, randomization and allocation of the study subjects. 
Demographic characteristics were comparable between the two groups (Table 1). Type of surgery, type of surgical position, mean propofol dose for induction of anesthesia, and intraoperative hemodynamic parameters were not significantly different between the two groups (Table 2). By the within-subjects effects test of RM-ANOVA, SPI values were not significantly different between six time points in each group (sevoflurane group: $P=0.087$; desflurane group: $P=0.601$ ). There was no occurrence of definite anesthesia awareness in any group. No complications associated with ISBPB were noted.

By the between-subjects effects test of RM-ANOVA, SPI values throughout the entire study period were significantly higher in the sevoflurane group than in the desflurane group $(38.1 \pm 12.8$ vs. $30.7 \pm 8.8$, respectively, $P=0.005$ ). At all of the six time points, SPI values were significantly higher in the sevoflurane group than in the desflurane group (Figure 3). By the between-subjects effects test of RM-ANOVA, BIS values throughout the entire study period were significantly higher in the sevoflurane group than in the desflurane group ( $40.7 \pm 5.8$ vs. 36.8 \pm 6.2 , respectively, $P=0.008$ ). Mean BIS values at each time point, except T1 and T2, were significantly higher in the sevoflurane group than in the desflurane group (Figure 4).

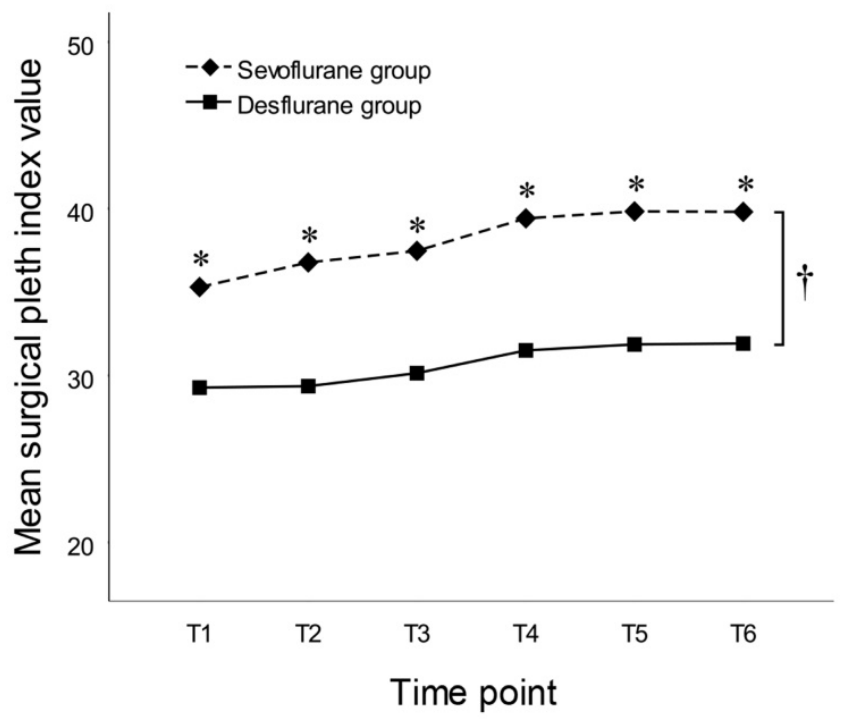

Figure 3. Time courses of mean surgical pleth index values in subjects anesthetized with sevoflurane or desflurane of 1.0 MAC. Time points: TI (sterile drape); T2 (portal insertion); T3 (synovectomy and debridement); T4 (acromioplasty); T5 (tendon repair); and T6 (wound closure). ${ }^{*} P<0.05$ by Student's $t$-test at each time point between groups. $+P=0.005$ by the between-subjects effects test of repeated-measures ANOVA throughout the entire study period between groups.
Table 1. Demographic characteristics of subjects

\begin{tabular}{llll}
\hline & $\begin{array}{l}\text { Sevoflurane group } \\
(n=36)\end{array}$ & $\begin{array}{l}\text { Desflurane group } \\
(n=36)\end{array}$ & $P$ value \\
\hline Age (years) & $52.4 \pm 11.8$ & $53.4 \pm 9.2$ & 0.690 \\
Sex (male/female) & $19 / 17$ & $20 / 16$ & 0.813 \\
Height $(\mathrm{cm})$ & $163.3 \pm 11.0$ & $163.9 \pm 8.3$ & 0.817 \\
Weight $(\mathrm{kg})$ & $65.5 \pm 10.6$ & $66.0 \pm 13.7$ & 0.876 \\
BMI $\left(\mathrm{kg} / \mathrm{m}^{2}\right)$ & $24.5 \pm 3.0$ & $24.4 \pm 3.6$ & 0.838 \\
ASAPS $(\mathrm{I} / \mathrm{II})$ & $17 / 19$ & $18 / 18$ & 0.814 \\
\hline
\end{tabular}

Data are expressed as the frequencies or means \pm SDs, as appropriate.

BMI: body mass index; ASAPS: American Society of Anaesthesiologists physical status.

Table 2. Clinical characteristics and haemodynamic parameters of subjects

\begin{tabular}{llll}
\hline & $\begin{array}{l}\text { Sevoflurane } \\
\text { group }(n=36)\end{array}$ & $\begin{array}{l}\text { Desflurane group } \\
(n=36)\end{array}$ & $P$ value \\
\hline $\begin{array}{l}\text { Type of surgery } \\
\text { Rotator cuff repair }\end{array}$ & 32 & 34 & 0.394 \\
$\begin{array}{l}\text { Capsular reconstruction } \\
\text { Type of surgical position }\end{array}$ & 4 & 2 & 0.257 \\
Lateral decubitus & 30 & 26 & \\
Beach chair & 6 & 10 & 0.480 \\
$\begin{array}{l}\text { Propofol dose }(\mathrm{mg}) \text { a } \\
\text { Haemodynamics b }\end{array}$ & $72.1 \pm 12.3$ & $74.6 \pm 16.6$ & \\
MAP (mmHg) & $81.5 \pm 12.4$ & $78.4 \pm 11.0$ & 0.247 \\
HR (beats/min) & $70.5 \pm 10.8$ & $68.6 \pm 9.6$ & 0.421 \\
\hline
\end{tabular}

Data are expressed as the frequencies or means \pm SDs, as appropriate.

MAP: mean arterial pressure; HR: heart rate.

a Bolus dose infused for induction of anaesthesia via target-controlled infusion device.

b Compared by Student's $t$-test using mean values throughout the entire study period. Haemodynamic parameters at each time point were also no significantly different between groups at any time point.

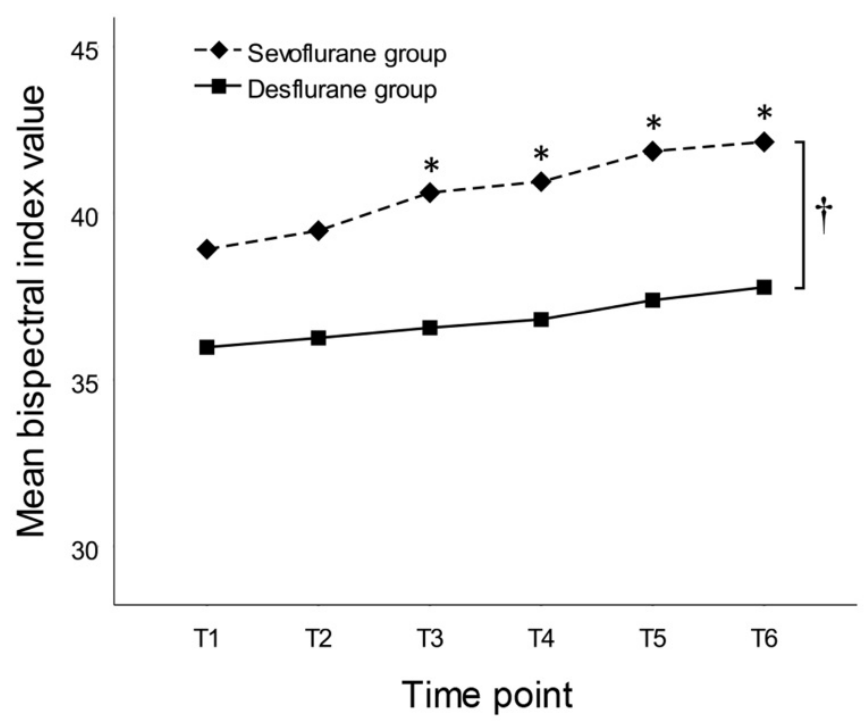

Figure 4. Time courses of mean bispectral index values in subjects anesthetized with sevoflurane or desflurane of 1.0 MAC. Time points: TI (sterile drape); T2 (portal insertion); T3 (synovectomy and debridement); T4 (acromioplasty); T5 (tendon repair); and T6 (wound closure). ${ }^{*} P<0.05$ by Student's $t$-test at each time point between groups. $+P=0.008$ by the between-subjects effects test of repeated-measures ANOVA throughout the entire study period between groups. 


\section{Discussion}

In this prospective, randomized, controlled study, we found that equi-MAC of different volatile anesthetics did not produce similar surgical pleth index values. Desflurane administered at equipotent 1.0 MAC produced significantly lower SPI values than sevoflurane.

Hypnosis, analgesia, and immobility are the three major components of general anesthesia [2, 19]. Ideal balanced anesthesia can be achieved using a combination of different anesthetic agents [20]. Most of the anesthetics act on the central nervous system as a whole, including the cortical (loss of consciousness) and subcortical (antinociception) brain areas and the spinal cord (muscle relaxation) [21]. Traditionally, MAC has been used as the standard measure to compare the potencies of volatile anesthetics. However, the MAC concept reflects the spinal mechanism of immobility rather than cerebral mechanism [22]. Volatile anesthetics cause immobility by spinal a-motor neuron depression [23]. Therefore, it is illogical to evaluate the level of hypnosis or analgesia, other major components of general anesthesia, using MAC. On the other hand, electroencephalogram (EEG)-derived variables, such as BIS, have been designed to reflect the level of consciousness rather than immobilization. Several studies have indicated that equi-MAC of various volatile anesthetics do not produce similar EEG-derived indices [3-8]. A study by Kim et al. suggested that the various effects of volatile anesthetics (i.e., hypnotic, analgesic and immobilizing effects) should be distinguished [7]. To date, however, there have been no controlled studies comparing the differences in analgesic properties of various volatile anesthetics at equi-MAC.

In general anesthesia, the levels of hypnosis and muscle relaxation are evaluated by a wide variety of monitoring devices, but there are no reliable tools to assess analgesia. In recent years, increasing numbers of reports have indicated that the SPI reflects nociception-antinociception balance during general anesthesia [12-16]. Huiku et al. reported that SPI value is high when noxious stimulation is high or remifentanil concentration inadequate, and that conversely, SPI value is low when remifentanil concentration is high or noxious stimulation is low [12]. Gruenewald et al. reported that the SPI response to a standardized tetanic stimulation was dependent on the remifentanil concentration during balanced anesthesia [14]. Chen et al. showed that the SPI values could predict the levels of stress hormones, such as adrenocorticotropic hormone, with high sensitivity and specificity [16]. In this study, SPI values were significantly lower with desflurane than with sevoflurane at a steady state of age-corrected 1.0 MAC. Based on previous studies and the findings presented here, we suggest that desflurane may have greater analgesic properties than sevoflurane at equipotent MAC.

The BIS is a multi-parameter EEG index with values ranging from 99 (awake) to 0 (isoelectric EEG), and is correlated with the level of hypnosis [24]. Volatile anesthetics produce dose-dependent effects on BIS $[25,26]$. In this study, the BIS values of the desflurane group were significantly lower than those of the sevoflurane group. This finding was consistent with the results of a previous study [7], which suggested that desflurane produces a greater hypnotic effect than sevoflurane during equipotent anesthesia.

In this study, there were no significant differences in BIS values at time points $\mathrm{T} 1$ and $\mathrm{T} 2$ between the two groups. The reason for these results is not clear, but there are a number of possible explanations. First, these observations may have been due to the residual hypnotic effects of propofol. To minimize the effects of propofol on the study outcomes, the minimum induction dose was used, an initial 30-minute waiting period before obtaining the first study data were allowed and all of the data were obtained after the $\mathrm{Ce}_{\text {propofol }}$ had decreased below 0.2 $\mu \mathrm{g} / \mathrm{ml}$. At the early time points of the study, however, $\mathrm{Ce}_{\text {propofol }}$ of about $0.2 \mu \mathrm{g} / \mathrm{ml}$ may have affected the BIS values $[27,28]$. Second, these results may have been due to the patient's surgical position, because two different surgical positions were used in this study (lateral decubitus or beach chair position). Changes in surgical position affect BIS values and may affect interpretation of the depth of anesthesia [29, 30]. Poorly controlled patient position may have affected BIS values, although there were no significant differences in the positions used between the groups (Table 2).

The application of ISBPB is a major strength of this study. ISBPB effectively controls the hemodynamic changes that occur during arthroscopic shoulder surgery as well as post-operative pain [31, 32]. In this study, to standardize intraoperative surgical stimulation, all of the subjects received ISBPB. Cases confirmed as a complete shoulder block in the pre- or post-operative test were included in the study. In both groups, there were no significant differences of SPI values between time points representing different surgical procedures. This finding means that, during the entire study period, the patients were not subjected to noxious surgical stimuli from the operative site as they only underwent homogenous non-specific stimulation, such as an irritation caused by the endotracheal tube and patient 
positioning device. This allowed general anesthesia to be maintained with only 1.0 MAC volatile anesthetic. The use of supplemental analgesics (e.g., remifentanil, nitrous oxide) can affect anesthetic depth measurement [33-37]. In our study, no other supplemental analgesics or hypnotics were administered throughout the study period. However, the application of ISBPB was also a limitation of the study. Due to the use of ISBPB, the SPI values were only obtained under non-surgical weak stimuli rather than painful surgical stimuli. Therefore, further studies using a standardized painful stimulus, such as long-lasting tetanic stimulation or laryngoscopic intubation, are needed to validate our results.

For several reasons, the results of this study need to be interpreted with caution. First, because SPI is a surrogate of the sympathetic response to noxious stimuli, different SPI profiles of sevoflurane and desflurane may be due to the direct impacts of volatile anesthetics on the autonomic nervous system rather than nociception-antinociception balance. Second, since hypnosis and analgesia, the major components of general anesthesia, is not completely distinct and interact with each other, it may be difficult to clearly distinguish the impacts of volatile anesthetics on SPI and BIS values, respectively. Another limitation of our study is that SPI and BIS profiles were only examined at a 'single' concentration of 1.0 MAC. Therefore, further investigations using different MAC values have to be conducted to validate our results.

In conclusion, desflurane showed greater analgesic properties with lower SPI values at equi-MAC compared to sevoflurane. Therefore, the equi-MAC of different volatile anesthetics does not guarantee similar analgesic properties.

\section{Abbreviations}

MAC: minimum alveolar concentration; SPI: surgical pleth index; BIS: bispectral index; ISBPB: interscalene brachial plexus block; TCI: target-controlled infusion; Ce: predicted effect-site concentration; MAP: mean arterial pressure; HR: heart rate; TOF: train-of-four; HBI: heart beat interval; PPGA: photoplethysmographic pulse wave amplitude; SD: standard deviation; IQR: interquartile range; BMI: body mass index; ASAPS: American Society of Anaesthesiologists physical status.

\section{Acknowledgments}

This research was supported by Basic Science Research Program through the National Research Foundation of Korea (NRF) funded by the Ministry of Science, ICT and future Planning (grant number: 2015R1A2A2A01006779, 2015). This study was supported by the National Research Foundation of
Korea (NRF) grant funded by the Korea government (NRF-2016R1A5A2012284).

\section{Competing Interests}

The authors have declared that no competing interest exists.

\section{References}

1. Aranake A, Mashour GA, Avidan MS. Minimum alveolar concentration: ongoing relevance and clinical utility. Anaesthesia. 2013; 68: 512-22.

2. Antognini JF, Carstens E. In vivo characterization of clinical anaesthesia and its components. Br J Anaesth. 2002; 89: 156-66.

3. Schwab HS, Seeberger MD, Eger EI, Kindler CH, Filipovic M. Sevoflurane decreases bispectral index values more than does halothane at equal MAC multiples. Anesth Analg. 2004; 99: 1723-7.

4. Umamaheswara Rao GS, Ali Z, Ramamoorthy M, Patil J. Equi-MAC concentrations of halothane and isoflurane do not produce similar bispectral index values. J Neurosurg Anesthesiol. 2007; 19: 93-6.

5. Taivainen T, Klockars J, Hiller A, Wennervirta J, van Gils MJ, Suominen P. The performance of Bispectral Index in children during equi-MAC halothane vs. sevoflurane anaesthesia. Eur J Anaesthesiol. 2008; 25: 933-9.

6. Prabhakar H, Ali Z, Bithal PK, Rath GP, Singh D, Dash HH. Isoflurane and sevoflurane decrease entropy indices more than halothane at equal MAC values. J Anesth. 2009; 23: 154-7.

7. Kim JK, Kim DK, Lee MJ. Relationship of bispectral index to minimum alveolar concentration during isoflurane, sevoflurane or desflurane anaesthesia. J Int Med Res. 2014; 42: 130-7.

8. Gupta M, Shri I, Sakia P, Govil D. Comparison of equi-minimum alveolar concentration of sevoflurane and isoflurane on bispectral index values during both wash in and wash out phases: A prospective randomised study. Indian J Anaesth. 2015; 59: 79-84.

9. Baik SW. Assessment of Depth of Anesthesia. Korean J Anesthesiol. 2007; 52: 253-61.

10. Crosby G, Culley DJ. Processed electroencephalogram and depth of anesthesia: window to nowhere or into the brain? Anesthesiology. 2012; 116: 235-7

11. Loughnan T, Loughnan AJ. Overview of the introduction of neuromuscular monitoring to clinical anaesthesia. Anaesthesia and intensive care. 2013; 41 Suppl 1: 19-24.

12. Huiku M, Uutela K, van Gils M, Korhonen I, Kymalainen M, Merilainen P, et al. Assessment of surgical stress during general anaesthesia. Br J Anaesth. 2007; 98: 447-55.

13. Struys MM, Vanpeteghem C, Huiku M, Uutela K, Blyaert NB, Mortier EP. Changes in a surgical stress index in response to standardized pain stimuli during propofol-remifentanil infusion. Br J Anaesth. 2007; 99: 359-67.

14. Gruenewald M, Meybohm P, Ilies C, Hocker J, Hanss R, Scholz J, et al. Influence of different remifentanil concentrations on the performance of the surgical stress index to detect a standardized painful stimulus during sevoflurane anaesthesia. Br J Anaesth. 2009; 103: 586-93.

15. Ledowski T, Pascoe E, Ang B, Schmarbeck T, Clarke MW, Fuller C, et al. Monitoring of intra-operative nociception: skin conductance and surgical stress index versus stress hormone plasma levels. Anaesthesia. 2010; 65: 1001-6.

16. Chen X, Thee C, Gruenewald M, Ilies C, Hocker J, Hanss R, et al. Correlation of surgical pleth index with stress hormones during propofol-remifentanil anaesthesia. ScientificWorldJournal. 2012; 2012: 879158

17. Eger EI, 2nd. Age, minimum alveolar anesthetic concentration, and minimum alveolar anesthetic concentration-awake. Anesth Analg. 2001; 93: 947-53.

18. Nickalls RW, Mapleson WW. Age-related iso-MAC charts for isoflurane, sevoflurane and desflurane in man. Br J Anaesth. 2003; 91: 170-4.

19. Gruenewald M, Ilies $\mathrm{C}$. Monitoring the nociception-anti-nociception balance. Best Pract Res Clin Anaesthesiol. 2013; 27: 235-47.

20. Tonner PH. Balanced anaesthesia today. Best Pract Res Clin Anaesthesiol. 2005; 19: 475-84.

21. Constant I, Sabourdin N. Monitoring depth of anesthesia: from consciousness to nociception. A window on subcortical brain activity. Paediatr Anaesth. $2015 ; 25: 73-82$

22. Rehberg B, Bouillon T, Zinserling J, Hoeft A. Comparative pharmacodynamic modeling of the electroencephalography-slowing effect of isoflurane, sevoflurane, and desflurane. Anesthesiology. 1999; 91: 397-405.

23. Rampil IJ, King BS. Volatile anesthetics depress spinal motor neurons. Anesthesiology. 1996; 85: 129-34.

24. Rampil IJ. A primer for EEG signal processing in anesthesia. Anesthesiology. 1998; 89: 980-1002.

25. Olofsen E, Dahan A. The dynamic relationship between end-tidal sevoflurane and isoflurane concentrations and bispectral index and spectral edge frequency of the electroencephalogram. Anesthesiology. 1999; 90: 1345-53.

26. Kreuer S, Bruhn J, Wilhelm W, Grundmann U, Rensing H, Ziegeler S. Comparative pharmacodynamic modeling of desflurane, sevoflurane and isoflurane. J Clin Monit Comput. 2009; 23: 299-305. 
27. Schüttler J, Ihmsen H. Population pharmacokinetics of propofol: a multicenter study. Anesthesiology. 2000; 92: 727-38.

28. Doi M, Gajraj RJ, Mantzaridis H, Kenny GN. Relationship between calculated blood concentration of propofol and electrophysiological variables during emergence from anaesthesia: comparison of bispectral index, spectral edge frequency, median frequency and auditory evoked potential index. $\mathrm{Br} \mathrm{J}$ Anaesth. 1997; 78: 180-4.

29. Kaki AM, Almarakbi WA. Does patient position influence the reading of the bispectral index monitor? Anesth Analg. 2009; 109: 1843-6.

30. Lee SW, Choi SE, Han JH, Park SW, Kang WJ, Choi YK. Effect of beach chair position on bispectral index values during arthroscopic shoulder surgery. Korean J Anesthesiol. 2014; 67: 235-9.

31. Singelyn FJ, Lhotel L, Fabre B. Pain relief after arthroscopic shoulder surgery: a comparison of intraarticular analgesia, suprascapular nerve block, and interscalene brachial plexus block. Anesth Analg. 2004; 99: 589-92.

32. Lee HY, Kim SH, So KY, Kim DJ. Effects of interscalene brachial plexus block to intra-operative hemodynamics and postoperative pain for arthroscopic shoulder surgery. Korean J Anesthesiol. 2012; 62: 30-4.

33. Heyse B, Proost JH, Hannivoort LN, Eleveld DJ, Luginbuhl M, Struys MM, et al. A response surface model approach for continuous measures of hypnotic and analgesic effect during sevoflurane-remifentanil interaction: quantifying the pharmacodynamic shift evoked by stimulation. Anesthesiology. 2014; 120: 1390-9.

34. Noh GJ, Kim KM, Jeong YB, Jeong SW, Yoon HS, Jeong SM, et al Electroencephalographic approximate entropy changes in healthy volunteers during remifentanil infusion. Anesthesiology. 2006; 104: 921-32.

35. Lee JH, Kim SI, Kim MG, Kim SC, Ok SY. The Influence of Remifentanil on the Bispectral Index during Intubation under TIVA using Propofol. Korean J Anesthesiol. 2007; 53: 695-9.

36. Nakayama M, Iwasaki S, Ichinose H, Yamamoto S, Satoh O, Nakabayashi K, et al. Effect of nitrous oxide on the bispectral index during sevoflurane anesthesia. Masui. 2002; 51: 973-6.

37. Ozcan MS, Ozcan MD, Khan QS, Thompson DM, Chetty PK. Does nitrous oxide affect bispectral index and state entropy when added to a propofol versus sevoflurane anesthetic? J Neurosurg Anesthesiol. 2010; 22: 309-15. 